\title{
Overview of Behaviour Characteristics of High and Low Literacy Users: Information Seeking of an Online Social Service System
}

\author{
Neesha Kodagoda, B.L. William Wong, and Nawaz Kahan \\ Interaction Design Centre \\ School of Computing Science, \\ Middlesex University, London, UK \\ $\{$ N. Kodagoda, W. Wong, N. Kahan $\}$ @mdx. ac.uk
}

\begin{abstract}
We investigated the differences in information seeking behaviours between low and high literacy users on an on-line social service system called the "Adviseguide" website. Ten volunteers participated in the study. Using the UK's National Skills for Life Survey, five were classified as high literate; five as low literate. Participants had four information search tasks that vary in difficulty: easy, medium and difficult. Observations, video recording, and a semi structured interview technique were used. The data were analysed using Grounded Theory and Emergent Themes Analysis approach. We identified eight information seeking behaviour characteristics; Reading/ Scanning, Focus, Satisfied, Verification, Recovery, Trajectories, Representation and Abandon. Results showed that low and high literacy users demonstrated critically different characteristics.
\end{abstract}

Keywords: Low \& high literacy users, information search task, information seeking behaviour characteristics.

\section{Introduction}

The purpose of this study is to investigate information seeking behaviours of low and high literacy users of an online social service system called "Adviceguide". This system is part of the services provided by the Barnet Citizens' Advice Bureau which provide support to clients from socially disadvantaged backgrounds. Literacy is the ability to read, write and count in one's primary language, taking advantages of the information sources available to them [1-3]. An adult can be categorised as a low literate when unable to read, write above UKs' national curriculum level 4. Literacy is measured using the National Skills for Life Survey which evaluates a person ability to read, write and listen and answer to questions or instructions. According to the UKs' National Skills for Life survey carried out in 2003, 16\% or equivalent to 5.2 million of the UK population presented low levels of literacy [4]. Research show low literacy users are more likely to be socially disadvantaged and also more likely to be unemployed $[4,5]$. 
A study by [6] show that low literacy users perform significantly worse than high literacy users as they were unable to find the information they were searching for or users assumed to have found the relevant information.

A previous study investigating low-literacy users reading strategies and navigational behaviour characteristics showed that low literacy users tend to read word by word, have a narrow field of focus, skip chunks of text if confronted by long and dense pages, quickly being satisfied with information found, minimise the amount of reading by skipping from one link to the another, and avoid searching as it requires spelling and typing [7].

As government and social services information are placed online, the problems faced by the low literacy users should be considered to enable digital inclusion. We analyse the information seeking behaviour characteristics between low and high literacy users of the "Adviceguide" website.

\section{Study}

An overview of the study is described below for more information about the experiment, participants, design, methodology and procedure please read [8].

Ten clients of the Citizens Advice Bureau volunteered for the study. They comprised six females and four males with an average age of 45 years. Using UK's National Skills for Life literacy assessment survey, five participants were classified as high literate, and the remaining five were classified as low literate. Low literacy participants showed a reading level below UKs' National Curriculum Level 4 while high literate users showed reading levels above 5. None of the participants had used the "Adviceguide" website previously, although all the participants had some experience in using the Internet.

Four information search tasks were developed based on the highest type of advice provided to the clients of the Barnet Citizens' Advice Bureau during April 2005 to May 2007. Information search task required the participant to find a specific piece of information, such as eligibility to receive benefits, money advice, assistance on giving up smoking, details of local child care availability, information on children hospital, and advice on council tax arrears. Users were refrained from using any internal or external search.

Multiple Cognitive Task Analysis (CTA) methods were used to extract and understand the human decision process during their cognitive work [9]. CTA methods used in our study were process tracing, observation and interview methods. Users were prevented from using the sites or other search facilities, users were asked to follow the web site menu links. The qualitative data were transcribed using HyperRESEARCH, and analysed using [10] Grounded Theory (GT) and [11] Emergent Themes Analysis (ETA) approach.

\section{Results}

Eight information seeking behaviour characteristics were identified: Verification, Reading/ Scanning, Recovery, Trajectories, Focus, Satisfied, Representation and Abandon. For detail results read [8]. 
Verification when users find information they need and examine other related links to support the information found for correctness. We did not observe this behavior in low literacy. For the purpose of this study, Reading behaviour took place when users read word by word, while Scanning behaviour referred to, users glancing through headings and subheadings or start, middle of a paragraph until they found something relevant or interesting. We observed low literacy users read word by word and that do not scan. Recovery refers to recuperate from a wrong or irrelevant information search to a more focused or relevant one resulting in finding the required information. We observed low literacy users were unable to recover from a mistake. The trajectories are information search paths taken by users. The paths taken by low literacy users were dissimilar. Focus when users are not likely to notice content above, below or to the side of their focus, results a narrow field of focus. We observed low literacy users had a narrow focus of attention. Satisfied as soon as the user assumes they have sufficient information and abandon the search task at an inappropriate place, due to being satisfied quickly. We observed low literacy users got satisfied with information quickly. Representation users' mental representation of information categories becomes a mismatch to system. We found low literacy users representations of information categories was a mismatch to the system. Abandon: when users show a higher tendency to give up their search due to many reasons. We found that low literacy users are likely to abandon an information search task.

\section{Discussion and Conclusion}

Our study was consistent with the findings of [7]. Both studies showed that low literacy users read word by word trying to make sense of information and do not present the ability to scan (reading). They have a narrow field of view and are not likely to notice content above, below or to the side of their focus (focus). They were likely to be satisfied and abandon the search early assuming they found relevant information (satisfied). However, we did not observed users tendency to skip chunks of text when faced with dense pages as described [7].

Our study found when users were presented with dense pages with anchor links, they were very likely to get lost and disoriented, this resulted low literacy users to abandon the search. We also identified the following characteristics: Low literacy users do not verify the information found for correctness (verification). They were unable to recover from a mistake even if they did identify wrong or irrelevant content (recovery). They did not share similar clues that lead to very different (trajectories) during their search paths. Low literacy users mental representations of the categories were a mismatch to the system (Representation). Finally, low literacy users had several reasons to abandon an information search task, (a) unable to find the information, (b) unable to recover from a mistake, (c) mental representation of the categories being a mismatch to the systems representation, (d) being satisfied quickly.

In conclusion, low literacy users demonstrated a critically different strategy from high literacy users when searching information using the "Adviceguide" website. They spend a lot of time reading instead of scanning, usually terminating the search before finding the right information. Verification was inexistent and a recurrent attitude to give up and terminate the search was presented. Their ability to recover 
from encountering wrong information was very low and they demonstrated a very narrow focus in all the cases. These behavioural patterns provoked low literacy users to use different search paths or trajectories.

These characteristics might help understand the low literacy user information seeking behaviour. Better interfaces design should help reduce low literacy users memory load, while maintain their attention by presenting less textual information; use high level linked clusters to afford rapid scanning so that they can see overall relationship structure; text which is simple to read; use of visual and audio where appropriate; assist users to recover from a search; try to match users mental perception with the interface design.

\section{References}

1. Chlebowska, K.: Literacy for Rural Women in the Third World. United Nations Educational Scientific and Cultural Organization (1990)

2. Baynham, M.: Literacy Practices, U.K (1995)

3. Stifelman, L.J., Schmandt, C., Hulteen, E.A.: VoiceNotes: A Speech Interface for a HandHeld Voice Notetaker. In: INTERCHI 1993 (1993)

4. Williams, J., et al.: The Skills for Life survey: A national needs and impact survey of literacy, numeracy and ICT skills. BMRB Social Research for the Department for education and Skills, London (2003)

5. Shalla, V., Schellenberg, G.: The Value of Words: Literacy and Economic Security in Canada. Catalogue No. 89-552-MPE. Statistics Canada, Ottawa (1998)

6. Kodagoda, N., Wong, B.L.W., Khan, N.: Effects of Low \& High Literacy on User Performance in Information Search and Retrieval. In: Proceedings of the 22nd annual conference of Interaction a specialist group of the BCS. HCI Liverpool 2008, vol. 1, pp. 173-181 (2008)

7. Summers, K., Summers, M.: Reading and Navigational Strategies Of Web Users With Lower Literacy Skills. 2005. In: 68th Annual Meeting Of The American Society For Information Science and Technology (ASIST) (2005)

8. Kodagoda, N., Wong, B.L.W., Khan, N.: Identifying Information Seeking Behaviours of Low and High Literacy Users: Combined Cognitive Task Analysis. In: NDM9 2009 (2009)

9. Wong, B.L.W.: The Critical Decision Method. In: International Encyclopaedia of Human Factors and Ergonomics, pp. 3067-3073. CRC Press, Boca Raton (2006)

10. Strauss, A., Corbin, J.: Basics of qualitative research: Grounded theory, procedures and techniques. Sage, Newbury Park (1998)

11. Wong, B.L.W., Blandford, A.: Analysing Ambulance Dispatcher Decision Making: Trialing Emergent Themes Analysis. In: HF 2002, Design for the whole person integrating physical, cognitive and social aspects. ESA \& CHISIG 2002 (2002) 